\title{
Isolation, Purification and Identification of a Novel HX-Producing Strain and Optimization of its Fermentation Medium
}

\author{
Xue $\mathbf{Z}^{1,2 *}$ and Zahng $\mathbf{S}^{3}$ \\ ${ }^{1}$ Luzhou Vocational and Technical College, Luzhou, PR China \\ ${ }^{2}$ Luzhou Biomedical Engineering Institute, Luzhou, PR China
}

${ }^{3}$ Luzhou Loajiao Group Co., Ltd, Sichuan Luzhou, PR China

\begin{abstract}
Hexanoic acid $(\mathrm{HX})$ is a crucial flavor compound and precursor of ethyl caproate (EA), which determines the quality of Chinese Luzhou-flavor liquor (CLFL). The isolation, purification, identification, and optimization of fermentation conditions of HX-producing bacteria are essential for industrial CLFL production. In this study, one strain of HX-producing bacterium was isolated from six candidate bacterial strains and identified as Clostridium sartagoneforme. Then, the growth characteristics and HX production of Clostridium sartagoneforme were investigated. Sodium acetate medium was identified as the optimal fermentation medium from four candidate media Clostridium sartagoneforme yielded $800.85 \pm 12.87 \mathrm{mg} / 100 \mathrm{~mL} \mathrm{HX}$ in sodium acetate medium. Then, to further optimize the formula of the fermentation medium, the carbon and nitrogen sources and inorganic salt component of the fermentation medium were investigated using $\mathrm{HX}$ yields as an optimization index. Optimization was performed with a single-factor experiment and the Taguchi design method. The single-factor experiment showed that the highest HX outputs were obtained when the sodium acetate medium contained $2.5 \mathrm{~g} / \mathrm{L}$ yeast extract, $1.8 \mathrm{~g} / \mathrm{LKCl}, 20 \mathrm{~g} / \mathrm{L}$ sodium acetate, $15 \mathrm{~mL} / \mathrm{L}$ ethanol, and $1.5 \mathrm{~g} / \mathrm{L}$ glucose. In the orthogonal experiment designed using the Taguchi design method, HX yields reached $2018.29 \pm 46.37 \mathrm{mg} / 100 \mathrm{~mL}$ in sodium acetate medium that contained $3.5 \mathrm{~g} / \mathrm{L}$ yeast extract, $1.8 \mathrm{~g} / \mathrm{L} \mathrm{KCl}, 25 \mathrm{~g} / \mathrm{L}$ sodium acetate, and $15 \mathrm{~mL} / \mathrm{L}$ ethanol.
\end{abstract}

Keywords: Isolation; Identification; Hexanoic acid-producing strain; Fermentation medium; Optimization

\section{Introduction}

Hexanoic acid (HX) is the precursor of ethyl caproate (EA), the main flavor component in Chinese Luzhou-flavor liquor (CLFL) according to the National Standard GB/T 10781.1-2006. EA is produced during the late fermentation stage of CLFL production in a mud pit, a fermentation container, by spirit reaction with HX. EA yields are determined by HX quantity from excessive spirits in lees, or fermented grains. The improvement of HX yields in CLFL production is a crucial technical problem for CLFL industries. Numerous methods have been developed to increase HX output in traditional liquor production [1].

One of the simplest and most widely adopted methods for improving CLFL quality is prolonged fermentation time. The fermentation time for regular liquor production is 45 days from lees entry to lees exit. To improve HX yield, the fermentation time for a pit in good fermentation condition is extended to 60,90 , or even 180 days. The relative HX amount, EA content, and CLFL quality increase as fermentation time increases. Another widely adopted method for improving CLFL production is multiple fermentation rounds (double fermentation). In this method, lees are retained in the bottom of the fermentation pit and are mixed with new fermentation starter powders (e.g., daqu in Chinese) for another one or two fermentation periods. After two or three fermentation periods, the amounts of HX and other organic acids increase and the microbial biochemical reaction of spirits with HX or other organic acids is enhanced, thus increasing total acid and ester contents and improving liquor flavor. The traditional methods to improve CLFL quality, however, decrease production efficiency and increase production cost [2].

For several centuries, the attempts of CLFL makers to economically improve liquor quality have been unsuccessful. A breakthrough in CLFL production finally occurred in the 1960s when EA was identified as the main flavor component of CLFL. The isolation, purification, and identification of HX-producing bacteria and the optimization of fermentation conditions are now routine work for the CLFL industry and researchers [3].
In the past decades, several HX-producing microbes have been isolated and identified from CLFL pit mud. The HX production of these bacterial strains outside of fermentation pits, however, are limited at $700 \mathrm{mg} / \mathrm{mL}$ HX [4].

In this study, we aimed to isolate and identify highly effective HXproducing bacteria. We also aimed to optimize fermentation conditions for the application of HX-producing bacteria in practical production.

\section{Materials and Methods}

\section{Materials}

Pit mud: The pit mud used in the present study was collected from the four corners and center of the bottom of a 180-year-old pit in Luzhou Laojiao Group Luzhou Laojiao Group Co. Ltd, a famous CLFL producer in Southern Sichuan, PRC.

Reagent: Yeast extracts, $\mathrm{KCl}$, glucose, sodium acetate, and absolute ethanol were purchased from Kelong Reagent Factory (China, Chengdu). The standard material 12-ethyl butyrate was purchased from Aladdin Reagent Co., Ltd (China, Shanghai).

\section{Culture medium}

Isolation and enrichment culture medium: The culture medium contained the following components:5g sodium acetate; $1 \mathrm{~g}$ yeast extracts; $10 \mathrm{~mL} 4 \%$ potassium dihydrogen phosphate; 5

*Corresponding author: Xue Z, Luzhou Vocational and Technical College, Luzhou Biomedical Engineering Institute, PR China, Tel: 0512-68418888; E-mail: kent988@Izy.edu.cn

Received March 07, 2018; Accepted April 10, 2018; Published April 18, 2018

Citation: Xue Z, Zahng S (2018) Isolation, Purification and Identification of a Novel HX-Producing Strain and Optimization of its Fermentation Medium. J Food Ind Microbiol 4: 126. doi:10.4172/2572-4134.1000126

Copyright: (c) 2018 Xue Z, et al. This is an open-access article distributed under the terms of the Creative Commons Attribution License, which permits unrestricted use, distribution, and reproduction in any medium, provided the original author and source are credited. 
$\mathrm{mL} 2 \%$ magnesium sulfate; $20 \mathrm{~mL} 0.5 \%$ calcium sulfate; $10 \mathrm{~mL} 5 \%$ ammonium sulfate; and $1 \mathrm{~mL} 5 \mathrm{~m} \mathrm{~g} / \mathrm{L}$ biotin. The culture medium was brought to $1000 \mathrm{~mL}$ with tap water and its $p H$ was adjusted to 6.0-6.5. Then, $2 \%$ agar was added to solidify the medium before sterilization at $121^{\circ} \mathrm{C}$ for $30 \mathrm{~min}$ in an autoclave. Prior to inoculation, $20 \mathrm{~mL}$ absolute ethanol was added to the medium.

Fermentation medium: Four fermentation media were selected for screening HX-producing bacteria.

\section{Methods}

Isolation of HX-producing bacteria from pit mud: $\Rightarrow$ Pit mud $\Rightarrow$ Preparation of seed solution $\Rightarrow$ Enrichment culture Plate separation $\Rightarrow$ Slope inoculation $\Rightarrow$ Primary screening $\Rightarrow$ Rescreening Gas $\Rightarrow$ chromatography (GC) analysis $\Rightarrow$ Strain preservation.

Preparation of seed solution: A total of $1 \mathrm{~g}$ pit mud was added to 9 $\mathrm{mL}$ liquid isolation medium in a stoppered test tube. The tube was then placed in a $90^{\circ} \mathrm{C}$ water bath for $10 \mathrm{~min}$. After cooling, $150 \mu \mathrm{L}$ absolute ethanol was added to the tube. The seed solution was then cultured at $34^{\circ} \mathrm{C}$ and $0.08 \mathrm{MPa}$ in a vacuum incubator until gas was produced.

Enrichment culture: A total of $1 \mathrm{~mL}$ HX-producing seed solution was pipetted and added to $9 \mathrm{~mL}$ liquid isolation culture medium in a stoppered tube. The tube was then placed in a $90^{\circ} \mathrm{C}$ water bath for 10 min. After cooling to $70^{\circ} \mathrm{C}, 150 \mu \mathrm{L}$ absolute ethanol was added to the enrichment culture. The culture was then incubated under the abovementioned conditions for 15 days.

Plate separation: The tube of enrichment culture that produced the highest amount of gas was selected. A total of $10 \mu \mathrm{L}$ enriched culture medium was drawn from the selected tube, spread on solid culture medium, and incubated at $90^{\circ} \mathrm{C}$ in an incubator for $10 \mathrm{~min}$. Subsequently, 2\% (V/V) absolute ethanol was added to the incubated culture medium until the culture medium cooled down to $70^{\circ} \mathrm{C}$. The inoculated medium was incubated under the above conditions until the colonies of HX-producing bacteria appeared [5-9]

Slant culture: Colonies that possessed a regular morphology were selected and transferred to the surfaces of slant media and cultured in the above-mentioned conditions until the colonies of HX-producing bacteria appeared.

Preliminary screening: Colonies with entire edges and slightly raised and smoothly rounded tops were selected. A loopful of bacteria was picked up, placed in a drop of water on a slide, and stained by Gram staining. The stained bacteria were observed under microscopy. Motile, Gram-positive, rod-shaped bacterial cells with ellipsoid spores were selected for future screening.

Rescreening: Individual colonies from six selected clones were picked and inoculated into $5 \mathrm{~mL}$ isolation culture medium under the above conditions for 6 days. The bacteria were then transferred to 50 $\mathrm{mL}$ of fermentation culture medium under the above conditions for 15 days. GC was used to investigate 15-day-old fermentation broths to select the most efficient strain of HX-producing bacteria based on HX yields.

Pretreatment of fermented broth: The $\mathrm{pH}$ of $1 \mathrm{~mL}$ fermented esterifying liquid was adjusted to $\mathrm{pH} 3$ with $1 \mathrm{M}$ hydrochloric acid solution. The amount of hydrochloric acid solution required to adjust the solution $\mathrm{pH}$ to 3 was recorded.

A total of $10 \mu \mathrm{L}$ 2-ethyl butyrate, the internal standard solution, was added to $490 \mu \mathrm{L}$ fermented esterifying liquid. The mixture was vortexed vigorously. Then, the mixture was acidified to $\mathrm{pH} 3$ with $1 \mathrm{M}$ hydrochloric acid solution at half the volume of the $1 \mathrm{~mL}$ esterifying liquid. The acidified mixture was vortexed vigorously and then centrifuged for 10 minutes at $12,000 \mathrm{r} / \mathrm{min}$. The supernatant was drawn and filtered using a $0.22 \mu \mathrm{M}$ filter and stored at $4^{\circ} \mathrm{C}$ for future use.

Conditions of GC analysis: Chromatographic column: LZP-930 Liquor special analysis column (purchased from the Chinese Academy of Sciences, Lanzhou Institute of Chemical Physics).

Chromatographic conditions:

Detector temperature: $200^{\circ} \mathrm{C}$

Vaporizer temperature: $200^{\circ} \mathrm{C}$

Injection mode: Split ratio: 50:1

Column length: $25.0 \mathrm{~mm}$

Inner diameter: $0.32 \mathrm{~mm}$

Coating thickness: $1 \mu \mathrm{m}$

Programmed temperature: $65^{\circ} \mathrm{C} \Rightarrow 5 \mathrm{~min} \Rightarrow 3.5^{\circ} \mathrm{C} / \mathrm{min} \Rightarrow 145^{\circ} \mathrm{C}$

Duration of Analysis: 28 min.

\section{Identification}

Molecular identification: Molecular biological identification was conducted based on the study of Xue [4].

Characteristics of growth and HX-production: Thirty $15 \mathrm{~mL}$ anaerobic tubes were filled with $15 \mathrm{~mL}$ fermentation culture medium each and inoculated with the seed liquid of HX-producing bacteria at $10 \%$ proportion. The inoculated media were cultured in a vacuum incubator at $34^{\circ} \mathrm{C}$ and $0.08 \mathrm{MPa}$. Three anaerobic tubes were selected every 2 days for bacterial counts and HX analysis.

Strain preservation screening fermentation culture media for high yields of HX: Four fermentation culture media, as described by Xue [4] were screened for high HX output.

\section{Optimization of fermentation culture media}

Screening of carbon source, nitrogen source, and inorganic salt: A single-factor experiment was used to change only one nutrient for the fermentation culture medium, e.g., the sodium acetate medium mentioned in section 1.2.3 of the present paper. The amounts of carbon sources (glucose, starch, lactose, and sucrose), nitrogen sources (yeast extract, beef extract, peptone, ammonium chloride, ammonium sulfate, and urea), and inorganic salts $\left(\mathrm{KCl}, \mathrm{K}_{2} \mathrm{HPO}_{4}, \mathrm{KH}_{2} \mathrm{PO}_{4}, \mathrm{MgSO}_{4}, \mathrm{MnSO}_{4}\right.$, $\mathrm{CaCO}_{3}$ ) were the same as those in the screened fermentation medium.

Single-factor experiment: A single-factor experiment was performed to determine the dosage range of the main components of fermentation culture medium A e.g., glucose, yeast extracts (used instead of beef extract due to cost), $\mathrm{KCl}$, sodium acetate, and alcohol. The experimental concentration gradient was composed of two to three concentration levels that increased or decreased the concentrations of the components of the sodium acetate culture medium.

Optimization of fermentation culture medium by Taguchi design method: Based on the single-factor experiment, four ingredients yeast extract, sodium acetate, ethanol and $\mathrm{KCl}$ of the fermentation culture medium at three levels (Table 1) were selected for further optimization. A scheme for the orthogonal test (Table 2), which includes three factors and three levels, was designed using the Taguchi design method in Minitab 17. 
Citation: Xue Z, Zahng S (2018) Isolation, Purification and Identification of a Novel HX-Producing Strain and Optimization of its Fermentation Medium. J Food Ind Microbiol 4: 126. doi:10.4172/2572-4134.1000126

Page 3 of 9

\begin{tabular}{|c|c|c|c|c|}
\hline \multirow[t]{3}{*}{ Level } & \multicolumn{4}{|c|}{ Factor } \\
\hline & Yeast extract & $\mathrm{KCl}$ & Sodium acetate & Ethanol \\
\hline & \multicolumn{3}{|c|}{$g / L$} & $\mathrm{~mL} / \mathrm{L}$ \\
\hline 1 & 1.5 & 1.2 & 15 & 10 \\
\hline 2 & 2.5 & 1.8 & 20 & 15 \\
\hline 3 & 3.5 & 2.4 & 25 & 20 \\
\hline
\end{tabular}

Table 1: Factors and levels.

\begin{tabular}{|c|c|c|c|c|}
\hline \multirow[t]{2}{*}{ Group } & Yeast extract & $\mathrm{KCl}$ & Sodium acetate & \multirow{2}{*}{$\begin{array}{c}\text { Ethanol } \\
\mathrm{mL} / \mathrm{L}\end{array}$} \\
\hline & \multicolumn{3}{|c|}{$g / L$} & \\
\hline 1 & 1.5 & 1.2 & 15 & 10 \\
\hline 2 & 1.5 & 1.8 & 20 & 15 \\
\hline 3 & 1.5 & 2.4 & 25 & 20 \\
\hline 4 & 2.5 & 1.2 & 20 & 20 \\
\hline 5 & 2.5 & 1.8 & 25 & 10 \\
\hline 6 & 2.5 & 2.4 & 15 & 15 \\
\hline 7 & 3.5 & 1.2 & 25 & 15 \\
\hline 8 & 3.5 & 1.8 & 15 & 20 \\
\hline 9 & 3.5 & 2.4 & 20 & 10 \\
\hline
\end{tabular}

Table 2: Orthogonal test for $\mathrm{HX}$ yields.

a) Standard material

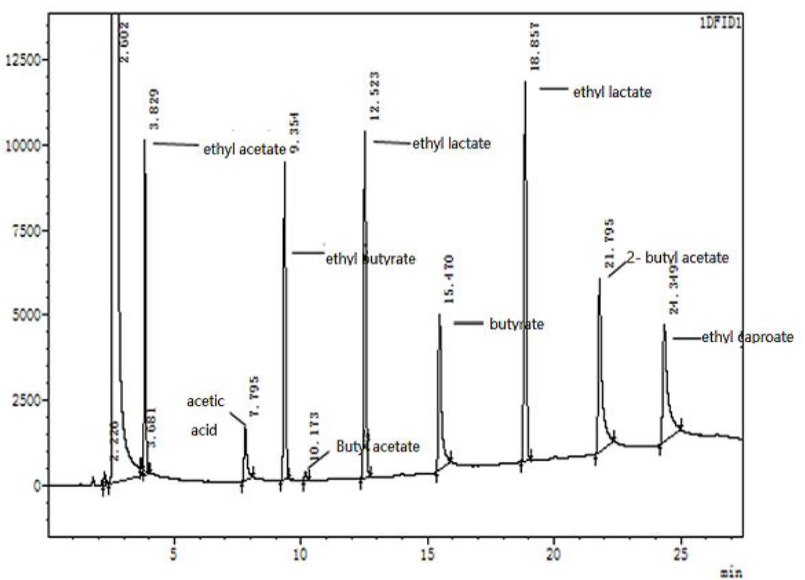

b) Fermentation broth

uV

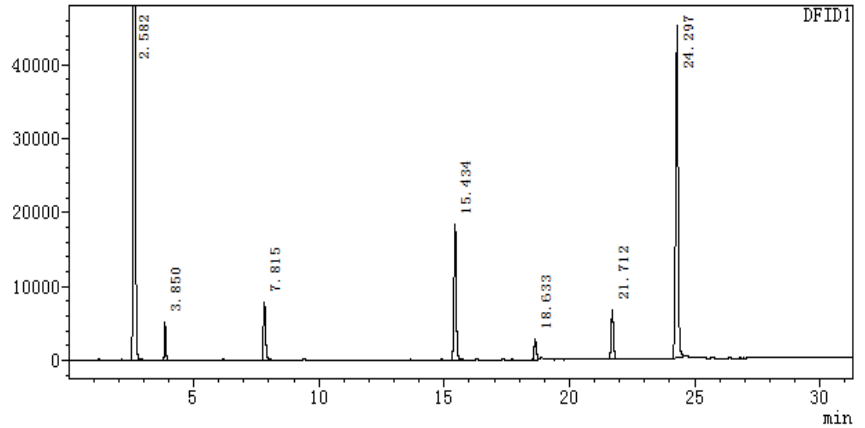

Figure 1: GC chromatograms.

The total cell number, which is represented by the optical density (OD) of 10 day fermentation broth, was detected by a spectrophotometer. The caproic acid content of the 15 day fermentation broth was detected using GC.

\section{Validation experiments}

Validation experiments were performed based on the optimal formula in section 1.2.4.3.

Data processing: Data were processed using Excel 2007 (Microsoft Excel, USA), Minitab 17 (PA, USA), and SPSS19 (Chicago, USA).

\section{Results and Discussion}

\section{GC analysis of fermented broth} broth.

The chromatographic peak in Figure 1 demonstrated that the GC conditions in the present study were suitable for HX detection.

The HX yields of six clones in 15 day fermented broth are shown in Table 3. The HX yields of six clones were compared with one-way ANOVA in SPSS 17. The results indicated that the HX yields of clone 5 in 15-dayfermented broth was significantly higher than those of clones $1,2,3,4$, and $6\left(\operatorname{Sig}_{\text {tow-tailed }}=0,0,0,0<0.01\right)$. Therefore, clone 5 was selected for investigation.

\section{Morphological characterization of clone 5}

The results for the morphological characterization of clone 5 are (Figure 2).

Clone 5 possesses the following characteristics:

1. Colony characteristics: round or roughly round; white or yellowish; opaque or translucent; relatively uniform edges; smooth surface; colony diameter 1.5-2 mm;

2. Bacterial cell morphology: long rods;

3. Spore morphology: ellipsoidal;

4. Spore-bearing position: mostly located in the center of the cell with a small number of cells at one end; 
Citation: Xue Z, Zahng S (2018) Isolation, Purification and Identification of a Novel HX-Producing Strain and Optimization of its Fermentation Medium. J Food Ind Microbiol 4: 126. doi:10.4172/2572-4134.1000126

Page 4 of 9

\begin{tabular}{|c|c|c|c|c|c|c|c|c|c|c|c|c|}
\hline Clones & & 1 & & 2 & & 3 & & 4 & & 5 & & 6 \\
\hline \multirow{3}{*}{$\begin{array}{c}\text { HX yields } \\
(\mathrm{mg} / 100 \mathrm{~mL})\end{array}$} & 189.56 & \multirow{3}{*}{$198.99 \pm 11$} & 234.76 & \multirow{3}{*}{$263.1 \pm 26.29$} & 206.33 & \multirow{3}{*}{$260.3 \pm 47.52$} & 365.89 & \multirow{3}{*}{$383.64 \pm 16.4$} & 585.78 & \multirow{3}{*}{$609.22 \pm 20.46$} & 167.34 & \multirow{3}{*}{$174.43 \pm 7.11$} \\
\hline & 196.35 & & 286.69 & & 295.89 & & 386.78 & & 623.45 & & 181.55 & \\
\hline & 211.07 & & 267.84 & & 278.67 & & 398.24 & & 618.44 & & 174.39 & \\
\hline
\end{tabular}

Table 3: HX yields of six clones.
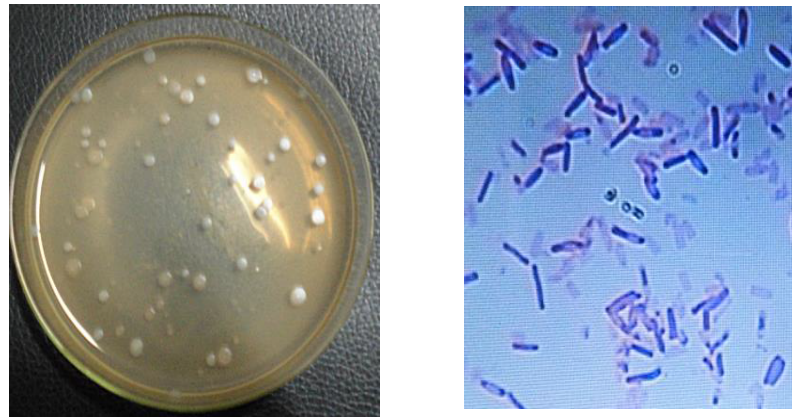

a) Colony characteristics of clone 5

b) Bacterial cell characteristics of clone $5(10 \times 100)$

Figure 2: Morphological characterization of clone 5.

5. Gram staining: $\mathrm{G}^{+}$.

\section{Molecular identification}

The gel electrophoresis results of 16SrDNA amplified by PCR were listed in Figure 3, the sequencing results for $16 \mathrm{SrDNA}$ fragments is listed in Figure 4 and the evolutionary tree constructed by Mega 7 was shown in Figure 4.

The sequence of the 16srDNA primer amplification product in Figure 3 was 1427-bp long, BLAST results in NCBI(https://blast.ncbi.nlm.nih. gov/Blast.cgi?PROGRAM=blastn\&PAGE_TYPE=BlastSearch\&LINK_ LOC=blasthome) revealed that clone 5 had a $100 \%$ sequence similarity to uncultured bacterium_claab17d12, uncultured bacterium clone aaa $62 \mathrm{~b} 11 \geq 99.5 \%$ to Clostridium sartagoneforme. A phylogenetic tree was constructed by selecting 100 strains of uncultured and cultural bacterial strains wit similarity $\geq 90 \%$ and the sequence of each strain to be tested. The strain of clone 5 was located in the same branch with the uncultured bacterium claab17d12 the similarity was $100 \%$ (Figure 4). According to the phylogenetic tree similarity combined with morphological characteristics, cultural characteristics, the strain was identified as Clostridium sartagoneforme, which is a cultural bacterium.

This is the first study to discover that C. sartagoneforme produces HX in CSFL pit mud.

\section{Growth curve and HX-production}

The results for HX-producing bacterial counts and HX contents are shown in Figure 5.

By analyzing the curves in Figure 5, the following conclusions were drawn:

1. Based on the bacterial growth curve, the initial lag period lasted for 4 days. The total number of bacterial cells (TNBC) negligibly increased during this period. The logarithmic growth phase occurred during the sixth to eighth days of fermentation. The TNBC increased the fastest during this period. The stationary phase occurred on the eighth to tenth days of fermentation. The TNBC negligibly increased during this period. Bacterial decay occurred from the 10th day to the end of fermentation. The highest TNBC was $53.72 \pm 1.32\left(\times 10^{7} \mathrm{cfu} /\right.$ $\mathrm{mL}$ ) and was observed on the $10^{\text {th }}$ day.

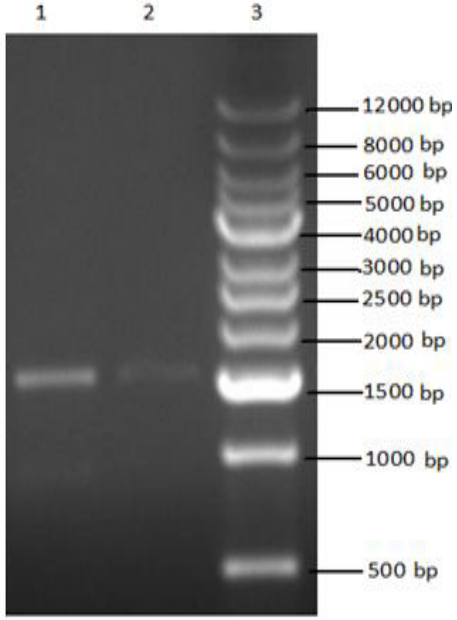

Figure 3: The 16srDNA of clone 5 .

2. Given that the fastest increase in HX production occurred during the eight to the $60^{\text {th }}$ day of fermentation, the optimal fermentation period for HX production is 16 days.

\section{Screening of HX-producing fermentation culture medium}

The results of GC analysis for HX yield in the four candidate fermentation culture media are shown in Table 4.

The results in Table4 revealed that the highest HX yield was in fermentation culture medium A. Further analysis by one-way ANOVA indicated that $\mathrm{HX}$ yield in fermentation culture medium $\mathrm{A}$ was significantly higher than those in fermentation culture media $\mathrm{B}, \mathrm{C}$, and D. Moreover, HX yield in fermentation culture medium A was higher than those in fermentation culture media B,C, and D by $4.07,3.90$, and 2.04 times, respectively.

\section{Screening for carbon source, nitrogen source, and inorganic salt}

HX yields in fermentation liquids with various carbon sources, nitrogen sources, and inorganic salts are shown in Figure 6. The following conclusions were drawn from the screening results for the optimal carbon source, nitrogen source, and inorganic salt for fermentation culture medium A:

1. Among the four carbon sources, the highest HX output 9 (Figure $6 \mathrm{~A}$ ) was achieved with $1 \mathrm{~g} / \mathrm{L}$ glucose as the carbon source. ANOVA analysis revealed that $\mathrm{HX}$ output with glucose as the carbon source was significantly higher $(\mathrm{P}<0.01)$ than those with lactose, sucrose, or starch as carbon sources.

2. Among the six nitrogen sources, the highest HX output (Figure $6 \mathrm{~B})$ was achieved with $2.5 \mathrm{~g} / \mathrm{L}$ beef extract as then nitrogen source. ANOVA analysis revealed that HX output in the fermentation liquid with beef extract as the nitrogen source was significantly higher than those with $(\mathrm{P}=0.048)$, ammonium sulfate, ammonium chloride, and urea $(\mathrm{P}<0.01)$ as nitrogen sources. HX yields with yeast or beef extracts 
Citation: Xue Z, Zahng S (2018) Isolation, Purification and Identification of a Novel HX-Producing Strain and Optimization of its Fermentation Medium. J Food Ind Microbiol 4: 126. doi:10.4172/2572-4134.1000126

TAAACATGCAAGTCGAGCGAGGAGATTCCCTTCGGGGATGAACCTAGCGGCGGACGGGTGAGTAACACGTGGGCAAC CTGCCTTATAGAGGGGAATAGCCTTCCGAAAGGAAGATTAATACCGCATAACATTACATTTTCGCATGAAGAAGTAATT AAAGGAGTAATCCGCTATAAGATGGGCCCGCGGCGCATTAGCTAGTTGGTGAGGTAACGGCTCACCAAGGCGACGATG CGTAGCCGACCTGAGAGGGTGATCGGCCACATTGGGACTGAGACACGGCCCAGACTCCTACGGGAGGCAGCAGTGG GGAATATTGCACAATGGGGGAAACCCTGATGCAGCAACGCCGCGTGAGTGATGAAGGTCTTCGGATCGTAAAGCTCTG TCTTCAGGGACGATAATGACGGTACCTGAGGAGGAAGCCACGGCTAACTACGTGCCAGCAGCCGCGGTAATACGTAGG TGGCGAGCGTTGTCCGGATTTACTGGGCGTAAAGGGAGCGTAGGCGGATTTTTAAGTGAGATGTGAAATACCCGGGCT CAACTTGGGTGCTGCATTTCAAACTGGAAGTCTAGAGTGCAGGAGAGGAGAGTGGAATTCCTAGTGTAGCGGTGAAA TGCGTAGAGATTAGGAAGAACACCAGTGGCGAAGGCGACTCTCTGGACTGTAACTGACGCTGAGGCTCGAAAGCGTG GGGAGCAAACAGGATTAGATACCCTGGTAGTCCACGCCGTAAACGATGAATACTAGGTGTAGGGGTTGTCATGACCTC TGTGCCGCCGCAAACGCATTAAGTATTCCGCCTGGGGAGTACGGTCGCAAGATTAAAACTCAAAGGAATTGACGGGG GCCCGCACAAGCAGCGGAGCATGTGGTTTAATTTGAAGCAACGCGAAGAACCTTACCTAGACTTGACATCTCCTGAAT TACTCTTAATCGAGGAAGTCCCTTCGGGGACAGGAAGACAGGTGGTGCATGGTTGTCGT-

CAGCTCGTGTCGTGAGATGTTGGGTTAAGTCCCGCAACGAGCGCAACCCTTATTGTTAGTTGCTACCATTAAGTTGAGC ACTCTAGCAAGACTGCCCGGGTTAACCGGGAGGAAGGTGGGGATGACGTCAAATCATCATGCCCCTTATGTCTAGGGC TACACACGTGCTACAATGGCAAGTACAAAAAGATGCAATACCGCAAGGTGGAGCTAAACTTAAAAACTTGTCTCAGTT CGGATTGTAGGCTGAAACTCGCCTACATGAAGCTGGAGTTGCTAGTAATCGCGAATCAGAATGTCGCGGTGAATACGTT CCCGGGCCTTGTACACACCGCCCGTCACACCATGAGAGTTGGCAATACCCAAAGTTCGTGAGCTAACCCGTAAGGGA GGCAGCGACCTAAGGTAGGGTCAGCGATTGGGGTGAAGTCGAACA

Figure 4: 16srDNA gene sequence.

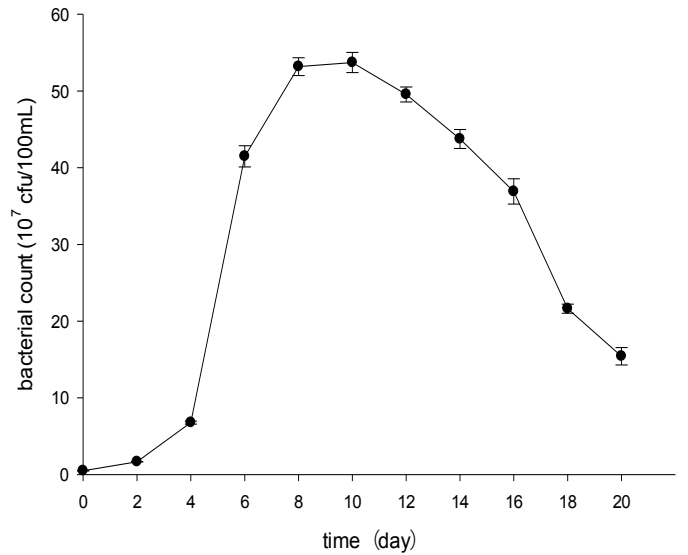

a) The growthcurve

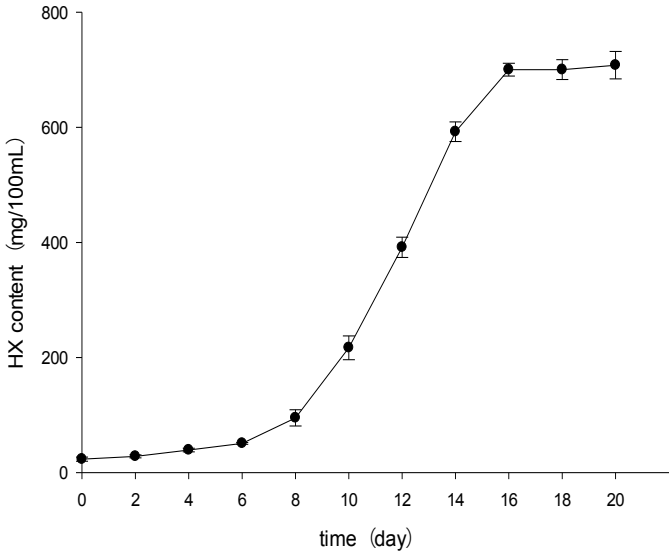

b) The HX-productioncurve

Figure 5: The growth and HX-production curves.

\begin{tabular}{|c|c|c|c|c|c|c|c|c|}
\hline Medium & \multicolumn{2}{|r|}{ A } & \multicolumn{2}{|c|}{ B } & \multicolumn{2}{|c|}{ C } & \multicolumn{2}{|r|}{ D } \\
\hline \multirow{3}{*}{$\begin{array}{c}\text { HX yields } \\
(\mathrm{mg} / 100 \mathrm{~mL})\end{array}$} & 788.726 & \multirow{3}{*}{$800.85 \pm 12.87$} & 189.110 & \multirow{3}{*}{$196.87 \pm 7.69$} & 194.150 & \multirow{3}{*}{$205.54 \pm 12.32$} & 374.65 & \multirow{3}{*}{$391.99 \pm 15.34$} \\
\hline & 814.352 & & 204.496 & & 203.847 & & 397.54 & \\
\hline & 799.46 & & 196.991 & & 218.617 & & 403.79 & \\
\hline
\end{tabular}

Table 4: $X$ yields in four candidate fermentation culture media.

as nitrogen sources were not significantly different $(\mathrm{P}=0.23)$. Therefore, yeast extract was used as the nitrogen source for further optimization because of economic reasons.

3. Among the inorganic salt sources, the highest HX output (Figure 6C) was achieved with $1.8 \mathrm{~g} / \mathrm{L} \mathrm{KCL}$. ANOVA analysis revealed that the HX output with KCL was significantly higher than all the others $(\mathrm{P}<0.01)$.
Therefore, the optimal carbon source, nitrogen source, and inorganic salt for $\mathrm{HX}$ production were glucose, beef extract, and $\mathrm{KCl}$.

\section{Single-factor experiment}

The HX contents in the 15-day fermentation liquid of the singlefactor experiment are shown in Figure 7.

The optimal concentration for each ingredient of the fermentation 

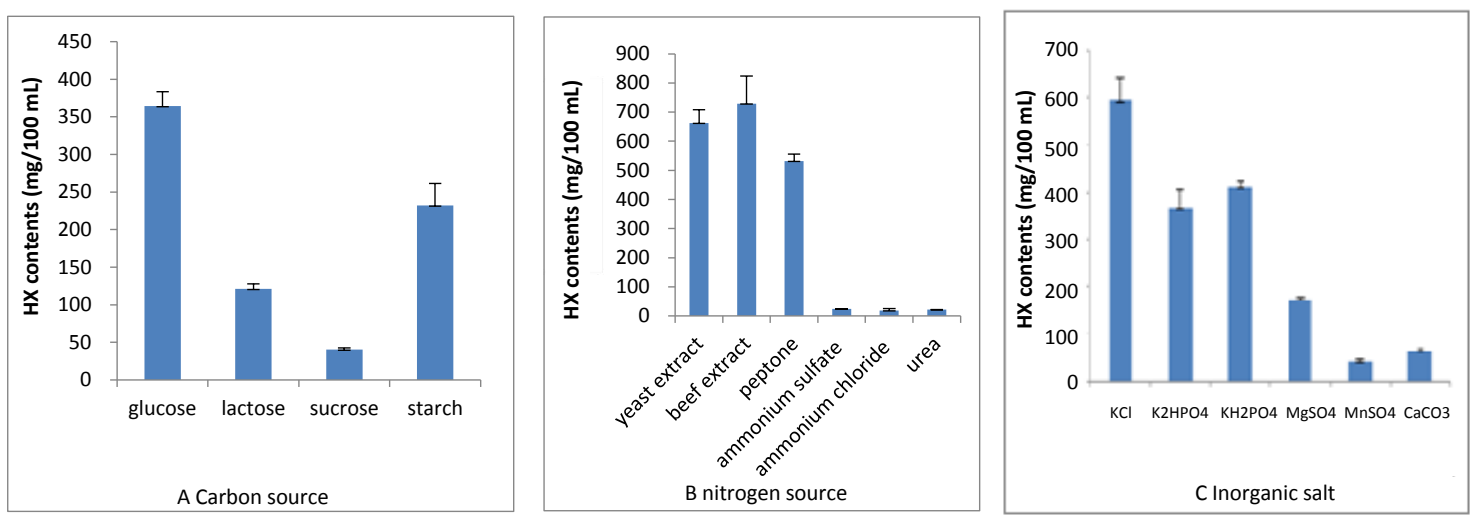

Figure 6: Screening results for carbon source $(A)$, nitrogen source $(B)$, and inorganic salt $(C)$.
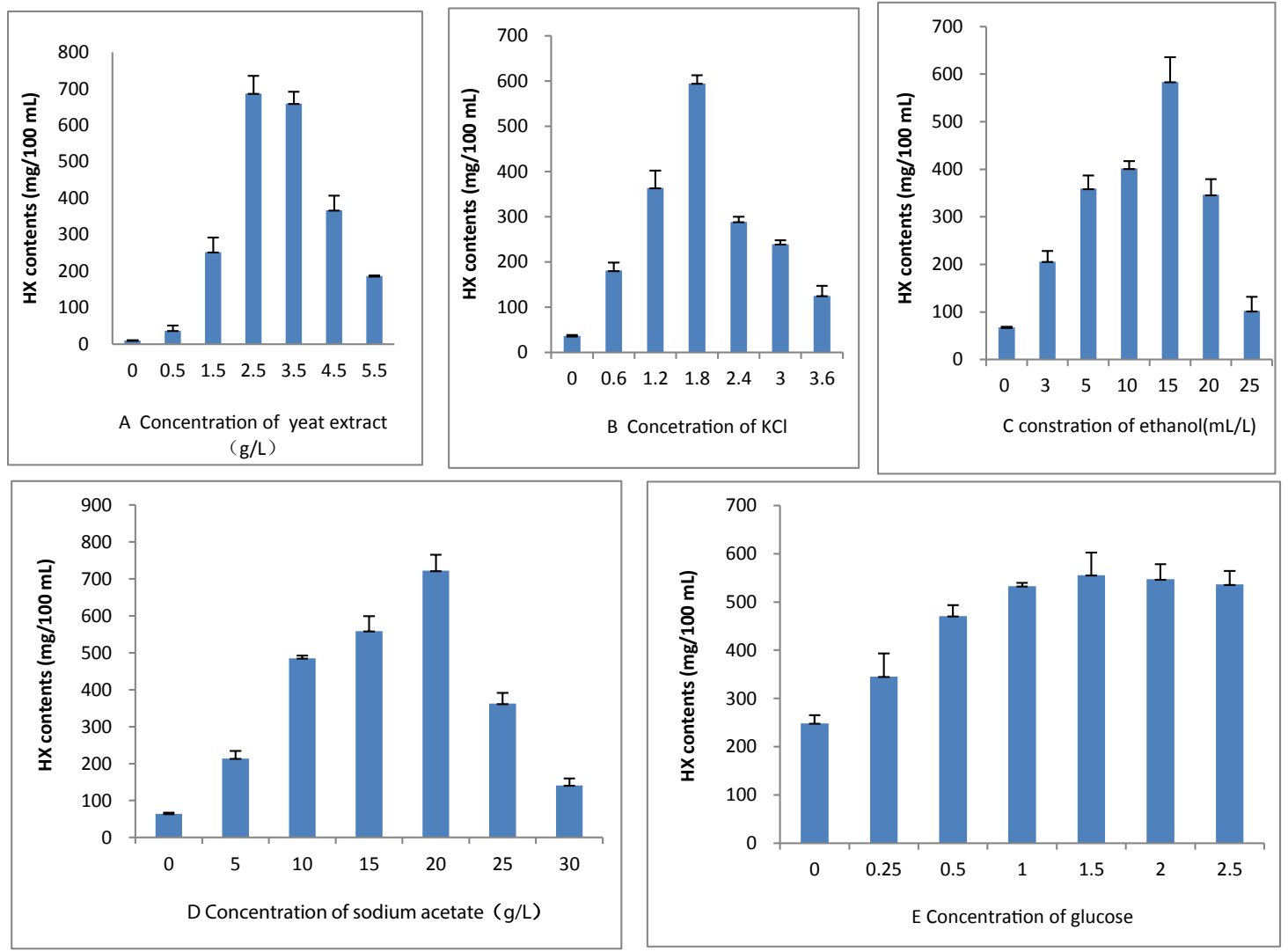

Figure 7: Results of single-factor experiment.

culture medium in HX production with strain $\mathrm{K} 3$ was $2.5 \mathrm{~g} / \mathrm{L}$ yeast extract, $1.8 \mathrm{~g} / \mathrm{L} \mathrm{KCl}, 15 \mathrm{~mL} / \mathrm{L}$ ethanol, $20 \mathrm{~g} / \mathrm{L}$ sodium acetate, and 1.5 $\mathrm{g} / \mathrm{L}$ glucose. The influence of each individual factor was calculated by subtracting the zero concentration from the highest HX production. Comparing the effects of individual factors revealed that sodium acetate was the most effective factor of HX production, whereas glucose was the most ineffective. Therefore, glucose was excluded from further optimization.

\section{Total cell number of fermentation broth by Taguchi design method}

A spectrophotometer was used to detect the total cell number, which was represented by OD, in 10-day fermentation broth that was optimized via the Taguchi design method. The results are shown in Figure 8.

The OD in Figure 8 demonstrated that group 1 had the highest cell number among all experimental groups. Comparing the OD in all the experimental groups revealed that the OD values of groups 1,2,6 and 9 were not significantly different $(\mathrm{P}>0.05)$. The $\mathrm{OD}$ values of these groups, however, are significantly higher than those of other groups $(\mathrm{P}<0.05)$.

\section{Optimization of HX production by Taguchi design method}

The GC detection results of the 16 day fermentation liquid are (Table 5). 
Citation: Xue Z, Zahng S (2018) Isolation, Purification and Identification of a Novel HX-Producing Strain and Optimization of its Fermentation Medium. J Food Ind Microbiol 4: 126. doi:10.4172/2572-4134.1000126

Page 7 of 9

Minitab 17 (Minitab Inc.; State College, PA) software was used to analyze the experimental results in Table 5 to obtain the response means of HX content, response means of SN ratios, mean main effect diagram, and the $\mathrm{SN}$ ratio of the main effect diagram. The location, dispersion, and adjustment factors were analytically determined to obtain the optimal factor and level combination for the fermentation medium [5]. The results were showed in (Tables 6 and 7), (Figures 9 and 10).

To identify the factors that significantly influence the mean value, the mean variance of the experimental results was analyzed further. The results are shown in Table 8 analysis of variance of mean value.
Based on the analysis of response for the means of HX content response means for signal-to-noise ratios (SNR); main effect diagram for means of HX content (Figure 9); main effects diagram for SN ratios of each factor (Figure 10) results of variance analysis, and with caproic acid yields as the index, yeast extract, $\mathrm{KCl}$, sodium acetate, and ethanol all significantly influence $\mathrm{HX}$ content. The relative importance of these factors was as follows: sodium acetate $>\mathrm{KCl}>$ ethanol $>$ yeast extract. The optimal factor and level combination for HX output was level 3 yeast extract, level $2 \mathrm{KCl}$, level 3 sodium acetate, and level 2 ethanol. Specifically, the optimal formula for maximum HX output was $3.5 \mathrm{~g} / \mathrm{L}$ yeast extract, $1.8 \mathrm{~g} / \mathrm{L} \mathrm{KCl}, 25 \mathrm{~g} / \mathrm{L}$ sodium acetate, and $15 \mathrm{~mL} / \mathrm{L}$ ethanol.

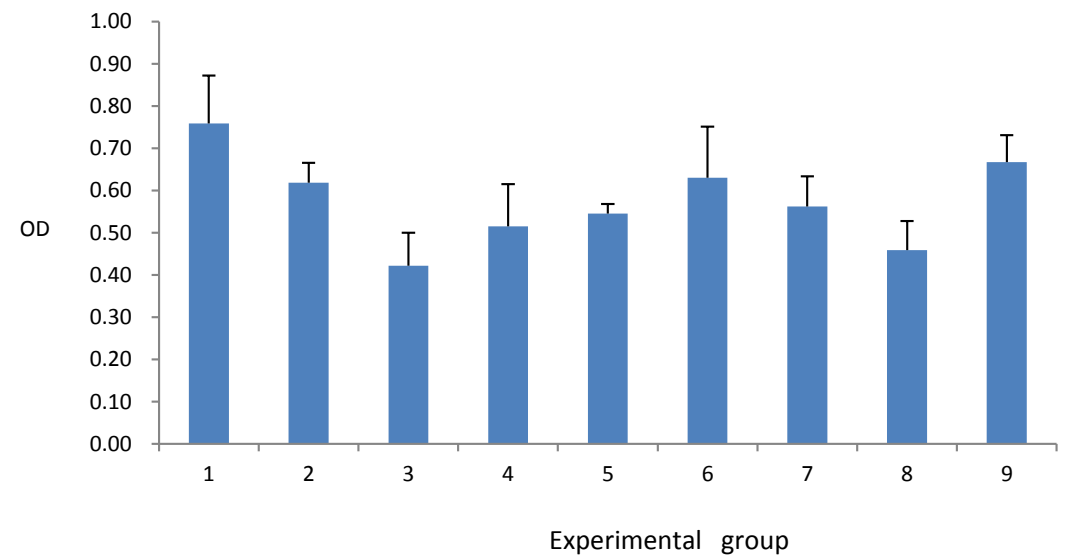

Figure 8: OD(optical density) of 10-day fermentation broth

\begin{tabular}{|c|c|c|c|c|c|c|c|c|}
\hline \multirow{2}{*}{$\begin{array}{c}\text { Test } \\
\text { number }\end{array}$} & \multicolumn{4}{|c|}{ Factors } & \multicolumn{3}{|c|}{ HX content $(\mathrm{mg} / 100 \mathrm{~mL})$} & \multirow{2}{*}{$\begin{array}{c}\mathrm{Y} \\
(\mathrm{mg} / 100 \mathrm{~mL})\end{array}$} \\
\hline & $\begin{array}{l}\text { Yeast extract } \\
(\mathrm{g} / \mathrm{L})\end{array}$ & $\begin{array}{c}\mathrm{KCl} \\
(\mathrm{g} / \mathrm{L})\end{array}$ & $\begin{array}{c}\text { Sodium acetate } \\
(\mathbf{g} / \mathbf{L})\end{array}$ & $\begin{array}{l}\text { Ethanol } \\
(\mathrm{mL} / \mathrm{L})\end{array}$ & 1 & 2 & 3 & \\
\hline 1 & 1.5 & 1.2 & 15 & 10 & 782 & 795 & 820 & 799 \\
\hline 2 & 1.5 & 1.8 & 20 & 15 & 1050 & 1026 & 1050 & 1042 \\
\hline 3 & 1.5 & 2.4 & 25 & 20 & 838 & 870 & 888 & 865.33 \\
\hline 4 & 2.5 & 1.2 & 20 & 20 & 717 & 730 & 697 & 714.67 \\
\hline 5 & 2.5 & 1.8 & 25 & 10 & 1169 & 1190 & 1269 & 1209.33 \\
\hline 6 & 2.5 & 2.4 & 15 & 15 & 671 & 680 & 691 & 680.67 \\
\hline 7 & 3.5 & 1.2 & 25 & 15 & 1283 & 1295 & 1383 & 1320.33 \\
\hline 8 & 3.5 & 1.8 & 15 & 20 & 828 & 830 & 858 & 838.67 \\
\hline 9 & 3.5 & 2.4 & 20 & 10 & 759 & 770 & 780 & 769.67 \\
\hline
\end{tabular}

Table 5: The HX content of the Taguchi design.

\begin{tabular}{|c|c|c|c|c|}
\hline \multirow{2}{*}{ Level } & \multicolumn{3}{|c|}{ Factors } \\
\cline { 2 - 4 } & Yeast extract & KCI & Sodium acetate \\
\hline 1 & 902.1 & 944.7 & 772.8 \\
\hline 2 & 868.2 & 1030.0 & 842.1 \\
\hline Delta & 976.2 & 771.9 & 1131.7 \\
\hline Rank & 108.0 & 258.1 & 358.9 & 1014.3 \\
\hline
\end{tabular}

Table 6: Response means for HX content.

\begin{tabular}{|c|c|c|c|}
\hline \multirow{2}{*}{ Level } & \multicolumn{3}{|c|}{ Factors } \\
\cline { 2 - 4 } & Yeast extract & KCl & Sodium acetate \\
\hline 1 & 59.05 & 59.18 & 57.73 \\
\hline 2 & 58.46 & 60.16 & 58.39 \\
\hline Delta & 59.54 & 57.71 & 60.94 \\
\hline Rank & 1.07 & 2.45 & 59.14 \\
\hline
\end{tabular}

Table 7: Response means for signal-to-noise ratios. 


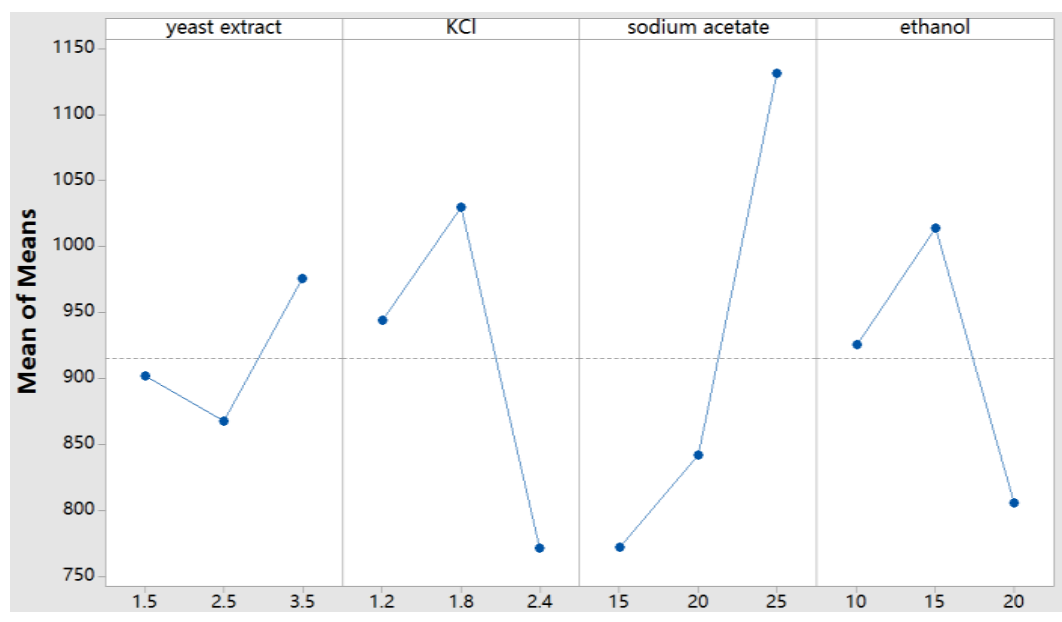

Figure 9: Main effects diagram of each factor for $\mathrm{HX}$ content.

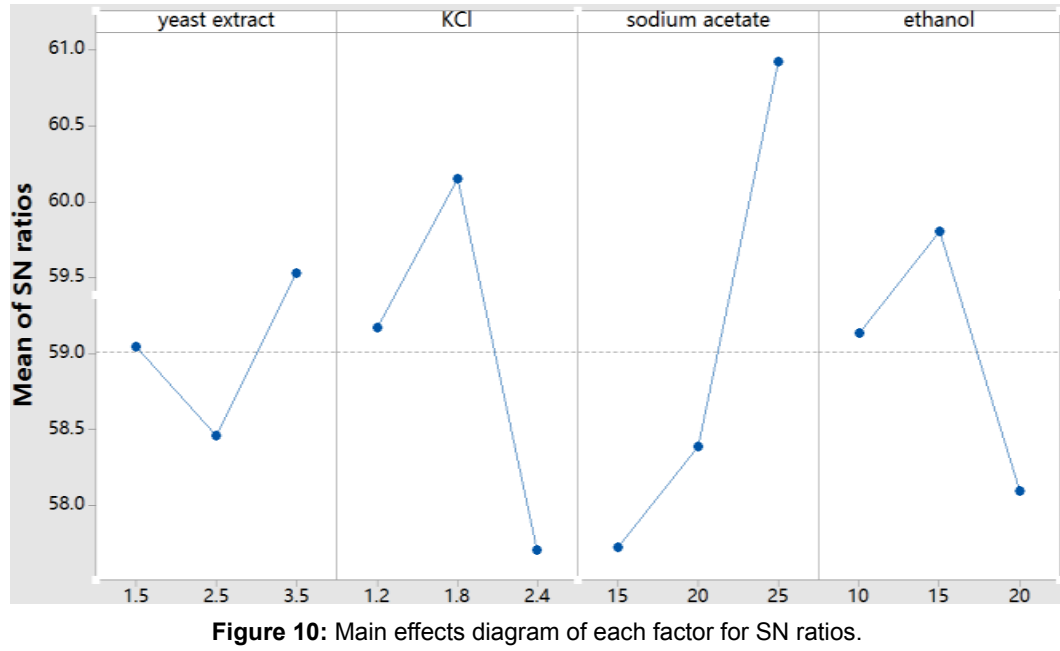

\begin{tabular}{|c|c|}
\hline Source & \\
\hline Yeast extract & \\
\hline $\mathrm{KCl}$ & \\
\hline Sodium acetate & \\
\hline Ethanol & \\
\hline Error & \\
\hline Total & \\
\hline
\end{tabular}

\begin{tabular}{|c|c|c|c|c|}
\hline DF & AdjSS & AdjMS & F-value & P-value \\
\hline 2 & 47115 & 23557 & 233.07 & 0 \\
\hline 2 & 288283 & 144142 & 1426.10 & 0 \\
\hline 2 & 592738 & 296369 & 2932.20 & 0 \\
\hline 18 & 173352 & 86676 & 857.55 & 0 \\
\hline 26 & 1819 & 101 & & \\
\hline
\end{tabular}

Table 8: Analysis of variance of mean value.

The predicted HX yield using this formula was $2112.346 \mathrm{mg} / 100 \mathrm{~mL}$.

\section{Validation experiment}

The mean of HX yields in the validation experiment was $2018.29 \pm$ $46.37 \mathrm{mg} / 100 \mathrm{~mL}$ and the SNR was8.78dB. The results demonstrated that the HX yields in the validation experiment were close to the predicted HX yield, thus indicating that this optimization scheme can achieve the desired effect and has good stability [10-12].

\section{Conclusion}

In the present study, an efficient HX-producing strain was isolated from 280 year old pit mud. This strain yielded 609.22 \pm 20.46 $\mathrm{mg} / 100 \mathrm{~mL}$ HX. Morphological identification indicated that the strain possessed the typical characteristics of HX-producing strains, such as $\mathrm{G}^{+}$and spore formation [12-14]. Bioinformatics analysis revealed that the strain shared $99.1 \%$ sequence similarity with $C$. sartagoneforme. Meanwhile, the four candidate fermentation culture media A, B, C and $\mathrm{D}$ were tested on the basis of maximum caproic acid yield. Singlefactor experiments were performed based on the selected fermentation culture medium A. The results of the single-factor tests showed that the maximum HX yields in 15-day-old fermentation broth were obtained with culture medium A that contained $2.5 \mathrm{~g} / \mathrm{L}$ yeast extract, $1.8 \mathrm{~g} / \mathrm{L}$ $\mathrm{KCl}, 20 \mathrm{~g} / \mathrm{L}$ sodium acetate, and $15 \mathrm{~mL} / \mathrm{L}$ ethanol. A further orthogonal experiment was designed using Taguchi methods in Minitab 17. The results demonstrated that the optimum formula for maximum HX output was $3.5 \mathrm{~g} / \mathrm{L}$ yeast extract, $1.8 \mathrm{~g} / \mathrm{L} \mathrm{KCl}, 25 \mathrm{~g} / \mathrm{L}$ sodium acetate, and 
Citation: Xue Z, Zahng S (2018) Isolation, Purification and Identification of a Novel HX-Producing Strain and Optimization of its Fermentation Medium. J Food Ind Microbiol 4: 126. doi:10.4172/2572-4134.1000126

Page 9 of 9

$15 \mathrm{~mL} / \mathrm{L}$ ethanol. The validation experiment had a maximum HX yield of $2018.29 \pm 46.37 \mathrm{mg} / 100 \mathrm{~mL}$.

\section{Acknowledgements}

This research was financially supported by the Science and Technology Support Plan Project of Sichuan Province (No.2014FZ0018), the Social Development Project of Luzhou Municipal Science and Technology Bureau(NO.2018-S-44(4/8)), and the Key Fund Project of Sichuan Provincial Department of Education(18ZA025).

\section{References}

1. Zhang FL (2008) Utilization of caproic acid bacteria pit mud for fermentation to improve the yield of kouzi liquor. Liquor-Making Sci Technol 22: 823-830.

2. Barros DPCD, Pinto F, Fonseca LP, Cabral JMS, Lemos F (2014) Kinetic model for the esterification of ethyl caproate for reaction optimization. $\mathrm{J}$ Mol Catal B Enzym 101: 16-22.

3. Cui HH and Cui JJ (2014) Isolation and purification of caproic acid bacteria from shilixiang aged pit mud and study of its expand training and application. Liquor Mak 41: 39-41.

4. Xue ZK (2014) Isolation and identification of a caproic acid-producing k-1 strain from luzhou-flavor liquor pit mud. J Proteomics 74: 2510-2521

5. Aminuddin NAB, Garzareyes JA, Kumar V, Antony J, Rochalona L (2016) An analysis of managerial factors affecting the implementation and use of overall equipment effectiveness. Int J Prod Res 54: 4430-4447.

6. Yang GR, Tang YT (2010) Culture of manmade pit mud \& its application. Liquor Mak 37: 25-27.
7. Xia PY, Xun-Liang SU, Xi-Wang XU (2005) Application of thaady in the production of manmade pit mud. Liquor-making Sci Technol, 136: 37-38.

8. Tan Z, Bao X, Du H, Chen Q, Han X, et al. (2014) Synthesis of ethyl caproate in $\mathrm{dbsa} /$ cyclohexane/water microemulsion system. J Chin I nst Food Sci Technol 14: 140-144.

9. Sun BG, Sun JY, Gong LL, Sun XT (2016) Perspectives on the long-term development trend and research emphasis of Chinese baijiu (Chinese liquor). J Light Ind 31: 6-11.

10. Maoke L, Yuming T, Ke Z, Daoqun R, Wanchun Y, et al. (2015) Analysis of action bacteria community and diversity in the pit mud of chinese luzhou-flavour liquor. Acta Ecologica Sinica 35(3)

11. Fan $W L$ and $X u Y(2014)$ Current practice and future trends of aroma and flavor of Chinese liquor (baijiu). J Food Saf Qual 3073-3078.

12. Chen Y, Luo W, Rui G, Xue X, Guan X, et al. (2016) Improved ethyl caproate production of chinese liquor yeast by overexpressing fatty acid synthesis genes with OPI1 deletion. J Ind Microbiol Biotechnol. 43: 1-10.

13. Hu XL, Du H, Xu Y (2015) Identification and quantification of the caproic acidproducing bacterium clostridium kluyveri, in the fermentation of pit mud used for chinese strong-aroma type liquor production. Int J Food Microbiol 214: 116-122.

14. Huang Z, Wei C, Bian M, Zong X, Luo H, et al. (2012) Isolation and identification of two bacteria strains from pit mud of luzhou-flavor liquor. Liquor-Making Sci Technol, 221: 36-38. 